\title{
Evolution of magnesium sulphate for eclampsia
}

\begin{abstract}
Eclampsia with its precursor preeclampsia is a condition that is rapidly taking the fore front in many countries as the leading cause of adverse maternal outcome, because of gains accruing from ability to curb, the better-understood obstetric haemorrhage. The exact mechanism by which preeclampsia leads to eclampsia remains unclear resulting in difficulty in prediction of individuals with preeclampsia who would develop eclampsia. Magnesium sulphate has been a recognised remedy since the early twentieth century, becoming scientifically proven and increasingly popular in the last two decades. However, the exact mechanism of action of magnesium sulphate in alleviating and preventing seizures still remains elusive and fits have been observed to occur during magnesium sulphate therapy. Better understanding of magnesium homeostasis in pregnancy and improved knowledge of the effects of magnesium sulphate may help unravel the mystery behind the cause and course of eclampsia.
\end{abstract}

Keywords: eclampsia, convulsion, pregnancy, magnesium sulphate, history, treatment, mortality

\author{
Special Issue - 2018
}

\author{
John Osaigbovoh Imaralu, Imaralu JO \\ Department of Obstetrics and Gynaecology, Babcock University, \\ Nigeria
}

Correspondence: John Osaigbovoh Imaralu, Department of Obstetrics and Gynaecology, Babcock University, llishan-Remo, Nigeria,Tel 2348067857419,Email imaraluj@gmail.com

Received: May 15, 2017 | Published: November 30, 2018

\section{Introduction}

Eclampsia is said to occur when a woman with preeclampsia experiences generalised tonic-clonic seizures during pregnancy or shortly after delivery. ${ }^{1}$ Eclampsia complicates between 1 to $2 \%$ of all cases of severe preeclampsia. ${ }^{2}$ Preeclampsia is a multisystemic disorder, characterised by hypertension and proteinuria occurring after the $20^{\text {th }}$ week of pregnancy in a woman who has been previously normotensive and non-proteinuric. It is classified as one of the hypertensive disorders of pregnancy. ${ }^{1,2}$ Eclampsia was first associated with albuminuria in 1839 , before hypertension in $1897 .{ }^{3}$ These discoveries, coupled with the introduction of antenatal care in the first decade of the $20^{\text {th }}$ century, led to better understanding and earlier diagnosis of the precursor condition known as preeclampsia. In spite of this, mortality from eclampsia still remained high in the 20th century, necessitating active search for treatment. ${ }^{3}$ The low and middle income countries suffer a disproportionately higher morbidity and mortality from preeclampsia and eclampsia. The incidence of eclamptic convulsions reported in developed countries is $1 / 2000$ while in developing countries; the incidence varies from 1/500 to $1 / 50$ deliveries. ${ }^{4}$ Most (99\%) of the 63,000 women worldwide that die every year from preeclampsia and eclampsia are in low-income countries. $^{5}$ The reported case fatality rates from eclampsia range from $1.8 \%$ in some centres in high-income countries, to as high as $15 \%$ in Nigeria and Bangladesh. ${ }^{6,7}$ This condition accounts for $25 \%$ of maternal deaths in Latin America and 10\% of maternal deaths in Africa and Asia. ${ }^{8}$

Preeclampsia can still be referred to as a disease of theories; as the exact course of events that lead to the clinical syndrome have not been fully determined. ${ }^{2}$ Similarly, the mechanisms by which seizures complicate preeclampsia, still remains unclear as eclampsia still happens suddenly today in women without overt features of severe preeclampsia 'like a flash of lightening' as coined by Veradeus.' Over the years, different anticonvulsants have been used, including magnesium sulphate $\left(\mathrm{MgSO}_{4}\right)$, phenytoin, diazepam, and "lytic cocktail" (usually chlorpromazine, promethazine, and pethidine). ${ }^{10}$ Magnesium sulphate has been in use for more than 80 years in the management of preeclampsia and eclampsia andit is today, the first choice for the prevention and the treatment of eclamptic convulsions. ${ }^{10}$ It has been shown in two large randomized control trials; the Collaborative Eclampsia Trial for women with eclampsia and the Magnesium sulfate for Prevention of Eclampsia (MAGPIE) Trial for women with preeclampsia, to be the most effective anticonvulsant for the prevention and treatment of seizures. ${ }^{3,11}$ In 2011, the WHO published evidence-based interventions for the prevention and treatment of preeclampsia and eclampsia; magnesium sulphate was recommended as the anticonvulsant of choice. ${ }^{12} \mathrm{~A}$ literature review on magnesium sulphate is thus imperative at this time, to look more closely at what is known now as we forge ahead probably towards the unknown for a better understanding of eclampsia.

\section{Discussion}

\section{The preeclampsia syndrome that results in eclampsia}

Today, Preeclampsia is still regarded as a disease of theories; as the exact course of events lead to it are yet to be fully determined. Although the exact aetiology remains elusive, two basic abnormalities have been consistently observed in preeclampsia; abnormal trophoblastic invasion of uterine blood vessels and endothelial cell dysfunction. ${ }^{13}$ It has been established that preeclampsia is fundamentally related to the failure of the second wave of trophoblastic invasion in the myometrium resulting in maternal spiral arterioles being hampered from becoming the normal physiologically high capacitance, low resistance vessels. ${ }^{3,10}$ Maternal endothelial cell dysfunction is corroborated by elevated serum level of endothelin-1, reduced level of nitric oxide (NO) and elevated level of vascular endothelial growth factor (VEGF). ${ }^{13}$ Other theories on the aetiology of preeclampsia include immunological, coagulation disturbance, placental factor and oxidative stress. ${ }^{13}$ Familial factor in the development of preeclampsia is suggested by a 3-4 fold increase in the incidence of preeclampsia in first degree relatives of affected woman, however the discordance in incidence between identical twins suggests other factors apart from the maternal genotype, raising the possibility of contribution from a fetal factor. ${ }^{14}$ 


\section{Mechanisms of seizuresin preeclampsia}

The exact mechanism by which preeclamptic women develop seizures is not known. Two hypotheses regarding the progression from preeclampsia to eclampsia have however gained prominence. These include the theory of cerebral ischemic necrosis, as evidenced by computed tomography $(\mathrm{CT})$ and magnetic resonance imaging (MRI) findings of areas of vasospasm, which may have caused necrosis. ${ }^{15}$ The neurological symptoms and imaging findings of eclampsia have been noted to be completely reversible in most cases, thus questioning ischemic necrosis as the mechanism of eclampsia occurrence. ${ }^{16}$ The second mechanism of occurrence of eclampsia is edema formation, which is of vasogenic origin resulting from a rapid rise in blood pressure that overcomes the myogenic vasoconstriction of cerebral arteries and arterioles. ${ }^{17}$ Eclampsia can thus be considered as a form of hypertensive encephalopathy, a condition also referred to as posterior reversible encephalopathy syndrome (PRES). ${ }^{16}$ This theory appears to have greater link as neuroimaging findings are consistent with cerebral oedema. The reversibility of headache, persistent vomiting, cortical blindness and seizures, after giving anti-hypertensive drugs are all explained by this theory. ${ }^{16}$

\section{Brief Historical overview of eclampsia treatment}

Eclampsia was distinguished from epilepsy in the $18^{\text {th }}$ century and was thought to be one of the pregnancy toxemias in which a circulating toxin acted on the "nerve centers to cause seizures. ${ }^{18}$ " Thus, popular treatment for eclampsia at that time involved toxin-eliminative therapy, such as phlebotomy, gastric lavage and carthasis. Later on, convulsions were thought to result in the disruption of the functioning of the heart, lungs, kidneys, and liver. Sedation became popular at this time with especially the work of Stroganoff who introduced morphine and chloral hydrate to decrease the frequency of convulsions. Oxygen was used to correct respiratory dysfunction and digitalis administered whenever the maternal pulse was rapid and weak after a seizure to restore cardiac function. ${ }^{3,18}$

\section{Magnesium levels in normal and preeclamptic preg- nancies}

The magnesium $(\mathrm{Mg}++)$ cation is an important cofactor for enzymatic reactions because of its important role in neuro-chemical transmission and muscular excitability. Only about $1-2 \%$ of total body magnesium exists in the extracellular space, $30 \%$ of which is bound to albumin. ${ }^{19}$ Normal plasma magnesium levels range from 1.5 to 2.5 $\mathrm{mEq} / \mathrm{L} .{ }^{19}$ Magnesium deficiency manifests as neurological symptoms, such as, increased muscle excitability, seizures and tremors. Hypocalcemia and hypokalemia can also follow low serum magnesium levels. Although a large store of magnesium exists intra-cellularly in adult bones, these stores are often poorly mobilized to maintain plasma levels. Parenteral magnesium therapy corrects the plasma deficit and stops deficiency symptoms and signs. ${ }^{19}$ Studies have shown that serum levels of magnesium are lower throughout normal uncomplicated pregnancy compared to the non-pregnant state. ${ }^{20}$

Serum levels of magnesium decreases progressively throughout pregnancy, such that hypomagnasemia predominates in the $2^{\text {nd }}$ and $3^{\text {rd }}$ trimesters. These changes are reversed at delivery, especially within the first 24 hours, when serum magnesium returns to the pre-pregnancy levels. ${ }^{21}$ Magnesium crosses the placental barrier and is transferred to the fetus through a trans-cellular route using the $\mathrm{Na}+/ \mathrm{Mg}^{2}+$ exchanger. A causal relationship may exist between serum magnesium levels and eclampsia since magnesium acts through intracellular inhibition of nitrous oxide (NO) synthase in endothelial cells to control blood pressure. ${ }^{19}$ Low serum magnesium levels have been consistently associated with occurrence of seizures in preeclamptics. ${ }^{20,22-24}$ As technological advances now allow for ionized magnesium to be more easily measured, questions have arisen as to whether it is more appropriate to monitor total serum magnesium, which is cheaper to do, or the ionized, physiologically active, form. In a study on preeclamptic patients that received $4 \mathrm{~g}$ intravenous loading dose followed by hourly $2 \mathrm{~g}$ infusions of magnesium sulphate, it was observed that both total and ionized $\mathrm{Mg}^{+2}$ concentrations increased rapidly after infusion, but steady-state concentrations for total magnesium were $4.84 \pm 0.24 \mathrm{mg} /$ $\mathrm{dL}$, whereas for ionized magnesium it was $2.04 \pm 0.14 \mathrm{mg} / \mathrm{Dl} .^{25}$ The routine assay of serum magnesium levels for assessing toxicity during magnesium sulphate therapy has been questioned by some authors. ${ }^{24,26}$

\section{Magnesium sulphate}

Successful treatment of spasms of tetanus with intra-thecal magnesium sulphate was the impetus for its intravenous trial in women with eclampsia in the early $20^{\text {th }}$ century. ${ }^{27}$ It was on this basis that an intern at the Los Angeles General Hospital in 1924, suggested intravenous magnesium sulphate for the treatment of eclampsia. The intravenous magnesium sulphate controlled the seizures in all 17 eclamptic women that received it and the observed maternal mortality rate of $6 \%$ was much lower than the existing historical average of $30 \% .^{28}$ Magnesium sulphate was later observed at the National level in the United States to reduce the mortality rate from preeclampsia to less than $5 \% .{ }^{29}$ Magnesium sulphate has been proven to be superior to both diazepam and phenytoin for the prevention of recurrent eclamptic seizures among 1700 women in the Collaborative eclampsia trial that included 23 centres from eight countries. ${ }^{10}$ More recently, in a comparison with placebo in the MAGPIE trial, magnesium cut both the risk of eclampsia and maternal mortality among 10,141 women with preeclampsia by more than half. ${ }^{28}$ There is now international consensus that magnesium is the treatment of choice for preeclampsia. ${ }^{12}$

Uses of magnesium sulphate: Magnesium sulphate in contemporary obstetric practice is administered to patients with severe preeclampsia or eclampsia. It is also used as tocolytic in the management of preterm labour. ${ }^{4,10}$ Administration of magnesium sulphate to patients with mild preeclampsia is however controversial, as it was observed in the MAGPIE trial, to reduce the incidence of convulsions by only $0.7 \%$ in the 7,468 women included in the study who did not have severe preeclampsia compared to placebo $(1.6 \%){ }^{28}$ The use of this drug in patients with pregnancy induced hypertension $(\mathrm{PIH})$ without proteinuria is also controversial. Proponents however continue to do so because about $25 \%$ of patients with PIH will develop pre-eclampsia and the lack of good predictive measures for women with preeclampsia who may develop seizures. ${ }^{30}$ The Potential benefits of administering magnesium sulphate to prevent or treat seizures at gestational ages less than 34 weeks and continuation till term may include; vasodilatation to improve blood flow in the pulmonary, renal, hepatic, central nervous system and placental circulations thereby delaying the need for delivery, this practice however remains debatable. ${ }^{10,22}$ The contraindications to magnesium therapy include; renal failure, myasthenia gravis and myocardial ischemia or failure, because of its depressive effect on respiratory and cardiac function. ${ }^{3,10}$

Pharmacology of magnesium sulphate: The exact mechanism of action of magnesium sulphate is not known, reports have it however that magnesium acts centrally to prevent convulsions by blocking 
neuromuscular transmission and decreasing the amount of acetylcholine liberated at the end plate by the motor nerve impulse. ${ }^{10,22}$ Although magnesium is known to exert a depressant effect on the central nervous system (CNS), it does not adversely affect the mother, fetus or neonate when the therapeutic plasma level is maintained during treatment of eclampsia. Magnesium acts peripherally to produce vasodilatation which may lead to flushing and sweating at low doses, while larger doses may cause a lowering of blood pressure. The central and peripheral effects of magnesium toxicity can be antagonized to by intravenously administration of calcium gluconate. ${ }^{22}$ As serum magnesium rises above the therapeutic range; the deep tendon reflexes first become depressed and then disappear as the level approaches 10 $\mathrm{mEq} / \mathrm{L}$. At this level respiratory paralysis may occur. Heart block also may occur at this level of magnesium. Serum magnesium concentrations higher than $12 \mathrm{mEq} / \mathrm{L}$ could result in cardiac arrest and death. ${ }^{10,20,22}$ The protocols for administration of magnesium sulphate in eclampsia and preeclampsia treatment include the Pritchard regimen which combines the intravenous route with the intramuscular route. ${ }^{31}$ The Zuspan and Sibai protocols are totally intravenous. ${ }^{10,22}$ Typical treatment duration are timed against the occurrence of fits and does not exceed 24 hours if when no further fits occur. ${ }^{10,22}$ Shorter regimens have been used in some centres in a low income country with outcomes comparable to that of the standard protocols. ${ }^{24,32}$ The onset of anticonvulsant action is immediate with the intravenous route and lasts about 30 minutes. Intramuscular administration results in the onset of action of about one hour and persists for three to four hours. Effective anticonvulsant serum levels range from 2.5 to $7.5 \mathrm{mEq} / \mathrm{liter}$ (4.8 to $8.4 \mathrm{mg} / \mathrm{dL}$ ). ${ }^{31}$ Magnesium is excreted solely by the kidney at a rate determined by the plasma concentration of magnesium and the glomerular filtration rate. ${ }^{31}$

Orally administered magnesium cannot cause a significant rise in total serum magnesium, because the kidneys will excrete it in the urine, thus maintaining a tight control. It is used as a cathartic because of its ability to absorb water locally and distend the intestinal lumen with resultant increase in peristalsis. ${ }^{19}$ This explains why magnesium is not presently administered orally to prevent or stop convulsions.

Mortality from magnesium sulphate toxicity have been associated with patient transfers to busy units with lower staffing levels, chaotic environments and dynamic nursing assignments. ${ }^{33}$ Efforts geared towards reducing harm when administering magnesium sulphate should include increasing staff ratios to allow for proper monitoring. Health care staff who use magnesium sulphate should have regular updates on how overdosing occurs and measures to prevent morbidity and mortality. ${ }^{33}$ Other measures for preventing toxicity, include stocking and use of calcium gluconate with clear directions in all units. Infusion pumps are preferred to ordinary gravity-fed fluid giving sets for administration of magnesium sulphate infusions, intravenous fluid containing magnesium sulphate should be labeled and in areas where only intravenous regimen exist, the pre-mixed solutions should be used, in order to prevent errors. ${ }^{33}$ Monitoring is the key to prevention of toxicity, as close observation of the patient's clinical condition is very important, some patients may develop toxicity even at therapeutic serum magnesium levels. ${ }^{22}$ The respiratory rate should be 16 cycles per minute or more and the deep tendon reflex normal prior to initiating magnesium sulphate therapy. The urine output during treatment should be at least $25 \mathrm{ml} /$ hour; half the dose of the magnesium sulphate should be given if less than $100 \mathrm{mls}$ of urine is produced in 4 hours. ${ }^{22}$ The fetal heart rate should be closely monitored while treating the mother with magnesium sulphate. The parameters observed should be interpreted with caution. High fetal levels can impair fetal breathing movements, which may lower the biophysical scores in the absence of significant hypoxia. ${ }^{22}$

\section{Conclusion}

Eclampsia is an established complication of preeclampsia, but the exact mechanism of progression to this complication remains unclear. It is also difficult to determine the specific features of preeclampsia that predict eclampsia. Magnesium sulphate is the most effective remedy in the history of treatment of eclampsia, its exact mechanism of action remains elusive. The cause and course of eclampsia may lie in the understanding of magnesium homeostasis in pregnancy and in increased knowledge of the effects of magnesium sulphate.

\section{Acknowledgements}

The author expresses special thanks to Drs. Odugbemi and Adelowo for exciting discussions and questions that raised interest leading to the conceptualization of this article.

\section{Conflict of interest}

The author declares no conflict of interest.

\section{References}

1. Report of the National High Blood Pressure Education Program Working Group on High Blood Pressure in Pregnancy. Am J Obstet Gynecol. 2000;183(1):21-22.

2. Steegers EA, von Dadelszen P, Duvekot JJ, et al. Preeclampsia. The Lancet. 2010;376(9741):631-644.

3. Lucas MJ, Leveno KJ, Cunningham FG. A comparison of magnesium sulfate with phenytoin for the prevention of eclampsia. $N$ Engl J Med. 1995;333(4):201-205.

4. Andersgaard AB, Herbst A, Johansen M, et al. Eclampsia in Scandinavia: incidence, substandard care, and potentially preventable cases. Acta Obstet Gynecol Scand. 2006;85(8):929-936.

5. Langer A, Villar J, Tell K, et al. Reducing eclampsia-related deaths a call to action. Lancet. 2008;371(9614):705-706.

6. Katz V, Farmer RM, Kuller JA. Preeclampsia into eclampsia: Toward a new paradigm. Am J Obstet Gynecol. 2000;182(6):1389-1396.

7. Igberase GO, Ebeigbe PN. Eclampsia: ten-years of experience in a rural tertiary hospital in the Niger delta, Nigeria. $J$ Obstet Gynecol. 2006;26(5):414-417.

8. Khan KS, Wojdyla D, Say L, et al. WHO analysis of causes of maternal death: a systematic review. The Lancet. 2006;367(9516):1066-1074.

9. Hallak M. Hypertension in Pregnancy. High risk pregnancy management option. 2nd ed. USA: WB Saunders; 1999. p. 639-663.

10. Euser AG, Cipolla MJ. Magnesium sulfate for the treatment of eclampsia: a brief review. Stroke. 2009;40(4):1169-1175.

11. Eclampsia Trial Collaborative Group: Which anticonvulsant for women with eclampsia? Evidence from the collaborative eclampsia trial. Lancet. 1995;345(8963):1455-1463.

12. WHO Recommendations for Prevention and Treatment of preeclampsia and eclampsia. WHO. 2011.

13. Redman CING, Sacks GP, Sargent IL. Preeclampsia: An excessive maternal inflammatory response to pregnancy. Am J Obstet Gynecol. 1999;180(2 pt 1):499-506. 
14. Hubel CA. Oxidative stress in the pathogenesis of preeclampsia. Proc Soc ExpBiol Med. 1999;222(3):222-235.

15. Koch S, Rabinstein A, Falcone S, et al. Diffusion-weighted imaging shows cytotoxic and vasogenicedema in eclampsia. Am J Neurorad. 2001;22(6):1068-1070.

16. Servillo G, Striano P, Striano S, et al. Posterior reversible encephalopathy syndrome (PRES) in obstetric critically ill patients. Intensive Care Med. 2003;29(12):2323-2232.

17. Thomas SV. Neurologic aspects of eclampsia. J Neurol Sci. 1998;155(1):37-43.

18. Thadhani RI, Johnson RJ, Karumanchi SA. Hypertension during Pregnancy: A Disorder Begging for Pathophysiological Support. xHypertension. 2005;46(6):1250-1251.

19. Elin RJ. Magnesium Metabolism in health and disease. DM. 1998;34(4):161-218.

20. Sanders R, Konijnenberg A, Huijgen HJ, et al. Intracellular and extracellular, ionized and total magnesium in pre-eclampsia and uncomplicated pregnancy. ClinChem Lab Med. 1999;37(1):55-59.

21. Shakmatova EI, Osipova NA, Natochin YV. Changes in Osmolality and blood serum ion concentrations in pregnancy. Human Physiology. 2012;26(1):92-95.

22. Lu J, Nightingale $\mathrm{CH}$. Magnesium sulfate in eclampsia and preeclampsia: pharmacokinetic principles. Clin Pharmacokinet. 2000;38(4):305314 .

23. Imaralu JO, Badejoko OO, Loto OM, et al. Determinants of seizure occurrence in preeclampsia before commencing and during treatment with magnesium sulphate. Int J Reprod Contracept Obstet Gynecol. 2016;5(10):3304-3311
24. Ekele BA, Badung SL. Is serum magnesium estimate necessary in patients with eclampsia on magnesium sulphate? Afr J Reprod Health. 2005;9(1):128-132.

25. Dube L, Granry JC. The therapeutic use of magnesium in anesthesiology, intensive care and emergency medicine: A review. Can J Anesth. 1993;50(7):732-746.

26. Koos BJ, Purcelli KJ. Judicious use of magnesium sulphate for eclampsia. The Journal of Family Practice. 2003;15(6):38-56.

27. Greene MF. Magnesium sulphate for preeclampsia. $N$ Eng $J$ Med.2003;384:275-276.

28. The Magpie Trial Collaborative Group. Do women with pre-eclampsia, and their babies, benefit from magnesium sulphate? The Magpie Trial: a randomised placebo-controlled trial. Lancet. 2002;359:1877-1890.

29. Usta IM, Sibai BM. Emergent management of puerperal eclampsia. Obstet Gynecol Clin N Am. 1995;22(2):315-335.

30. Sibai BM, Spinato JA, Watson DL, et al. Effect of magnesium sulfate on electroencephalographic findings in preeclampsia. Obstet Gynecol. 1984;64(2):261-266.

31. Pritchard JA, Cunningham FG, Prichard SA. The Parklands Memorial Hospital protocol for treatment of eclampsia: evaluation of 245 cases. Am J obstet Gynecol. 1984;148(7):951-63.

32. Abdul MA, Ibrahim UN, Yusuf MD. Low dose Magnesium sulphate in the management of eclamptic fits: Randomised controlled trial. Arch Gynecol Obstet. 2013;287(1):43-6.

33. Simpson KR, Knox GE. Obstetrical accidents involving IV magnesium sulfate. Am J Maternal Child Nurs. 2004;29(3):161-171. 